\title{
Probing the nano-scale: The first contact of an impacting drop
}

\author{
E. Q. $\mathbf{L i}^{1}$, I. U. Vakarelski ${ }^{1}$ and S. T. Thoroddsen ${ }^{1} \dagger$ \\ ${ }^{1}$ Division of Physical Sciences and Engineering \& Clean Combustion Research Center, \\ King Abdullah University of Science and Technology (KAUST), \\ Thuwal, 23955-6900, Saudi Arabia
}

(Received $\mathrm{xx}$; revised $\mathrm{xx}$; accepted $\mathrm{xx}$ )

When a drop impacts a solid surface, the lubrication pressure in the air deforms its bottom into a dimple. This makes the initial contact with the substrate occur not at a point but along a ring, thereby entrapping a central disc of air. We use ultra-highspeed imaging, with 200 ns time resolution, to observe the structure of this first contact between the liquid and a smooth solid surface. For a water drop impacting onto regular glass we observe a ring of micro-bubbles, owing to multiple initial contacts just before the formation of the fully wetted outer section. These contacts are spaced by a few microns and quickly grow in size until they meet, thereby leaving behind a ring of microbubbles marking the original air-disc diameter. On the other hand, no micro-bubbles are left behind when the drop impacts onto molecularly smooth mica sheets. We thereby conclude that the localized contacts are due to nanometric roughness of the glass surface and the presence of the micro-bubbles can therefore distinguish between glass with 10 $\mathrm{nm}$ roughness from perfectly smooth glass. We contrast this entrapment topology with the initial contact of a drop impacting onto a film of extremely viscous immiscible liquid, where the initial contact appears continuous along the ring. Here an azimuthal instability occurs during the rapid contraction at the triple line, also leaving behind micro-bubbles. For low impact velocities the nature of the initial contact changes to one initiated by ruptures of a thin lubricating air film.

\section{Introduction}

The impact of a drop on a solid is fundamental in many natural and industrial processes. One important aspect of the deposition is the entrapment of a bubble under the center of the drop. It can have detrimental effect on the uniformity in coating processes and affect conduction or conductivity through the interface between a solidified drop and the substrate. This central bubble was first observed in snapshot photographs (Chandra \& Avedisian (1991), Thoroddsen \& Sakakibara (1998)), but Thoroddsen et al. (2005) used high-speed video to image the initial entrapment of the air-disk and modeled its subsequent contraction into the central bubble. They also identified a dark ring, which often marked the initial diameter of the air disk, proposing it was due to the trapping of micro-bubbles.

The rapid contraction of the air-disc can also leave a small drop at the center of the bubble, when the converging capillary waves touch the dry substrate. This drop was imaged through the bottom glass by Thoroddsen et al. (2005) and later verified by Lee et al. (2012) using X-rays.

Liu et al. (2013) and Driscoll \& Nagel (2011) used interferometry with approximately

$\dagger$ Email address for correspondence: sigurdur.thoroddsen@kaust.edu.sa 
$7 \mu$ s time-resolution to observe the compression of the air-disc for high impact velocities. Subsequently, Li \& Thoroddsen (2015) used interferometry with 200 ns time-resolution to capture the entire compression and rebounding of the air-disc.

The theoretical treatment of the air-cushioning, started by Smith et al. (2003), is based on balancing the viscous lubrication in the thin air layer and the rapid deceleration of the drop inertia. Korobkin et al. (2008) added two deformable surfaces and Hicks \& Purvis (2010) reformulated the above 2-D theories for the axisymmetric case, to allow quantitative comparison with theory. They predicted the initial radius of the air-disc $L_{o}$ at first contact, as (Hicks et al. (2012), Hicks \& Purvis (2013))

$$
L_{o}=3.8\left(\frac{4 \mu_{g}}{\rho_{\ell} V}\right)^{1 / 3} R^{2 / 3},
$$

where $\mu_{g}$ is the gas viscosity, $\rho_{\ell}$ is the liquid density, $V$ is the impact velocity of the drop and $R$ the drop radius. This formula is in perfect correspondence with the recent experiments of Li \& Thoroddsen (2015), if one uses the bottom radius of curvature of the drop $R_{b}$ for $R$. This is mandated by the significant shape oscillations of the large water drops used in those experiments.

Thoroddsen et al. (2005) measured the size of the central bubble for high impact velocities, but for low impact velocities, Bouwhuis et al. (2012) identified a local parameter, where the size of the entrapped bubble is maximum.

Mandre et al. (2009) investigated the compressibility of the air, which occurs for higher impact velocities. The amount of compression is determined by a compressibility factor

$$
\epsilon^{-1}=\frac{\left(R V^{7} \rho_{\ell}^{4} / \mu_{g}\right)^{1 / 3}}{P_{a t m}} .
$$

If $\epsilon^{-1}>1$ one expects significant compression of the air-disc. For adiabatic compression the 2-D theory of Mandre et al. (2009) predicts that the air-layer thickness at the center-line reduces as $H^{*} /\left(R S t^{2 / 3}\right) \sim \epsilon^{1 / 3}$, where, following recent nomenclature, $S t$ is the inverse of the conventional Stokes number, i.e. $S t=\mu_{g} /\left(\rho_{\ell} R V\right)$. The experiments in Li \& Thoroddsen (2015) showed empirically that $H^{*} /\left(R_{b} S t^{2 / 3}\right)=4.2 \epsilon^{0.40}$. They also showed that the radial motion $U$ of the kink at the minimum gap (see sketch in Fig. 1a) is in good agreement with the above theory, i.e. $U / V \sim S t^{-1 / 3}$, see also Duchemin \& Josserand (2011).

Mandre et al. (2009) and subsequent work by Mani et al. (2010) and Mandre \& Brenner (2012) suggested the intriguing possibility that an impacting drop could skate on a thin layer of air and splash without ever touching the solid surface. However, the entrapment of the central air disc and its subsequent contraction into a bubble (Thoroddsen et al. (2005)), mandates a contact with the solid surface. Even though the skating on a thin layer of air may have little bearing on splashing, it is important for rebounding of the drop for small impact velocities, which is observed even for hydrophilic surfaces (Kolinski et al. (2014), de Ruiter et al. (2014)). de Ruiter et al. (2014) used smooth glass surfaces to find the maximum impact velocity where rebound without contact can take place, characterized by Weber numbers $W e=\rho_{\ell} R V^{2} / \sigma$ below 4 . Where $\sigma$ is the surface tension. Mandre \& Brenner (2012) highlight the importance of surface roughness, formulating conditions when the minimum air-gap will reach nano-metric thickness and minuscule asperities could rupture the film.

Herein we apply the ultra-high-speed direct imaging to investigate the nature of the initial contact of the drop liquid with the solid surface. In particular we identify the mechanism behind the formation of the characteristic ring of micro-bubbles which conveniently marks the location of the initial contact. Thereby, our imaging reveals a 


\section{(a)}

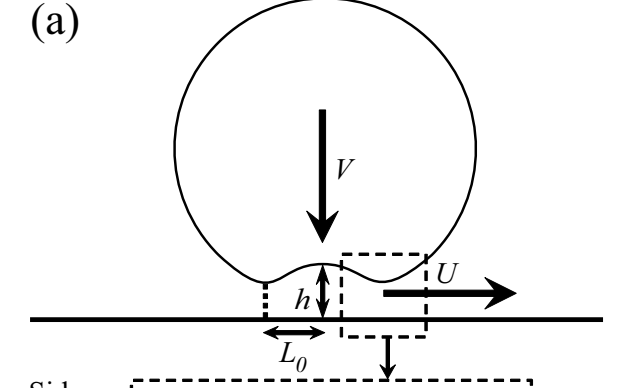

Side view

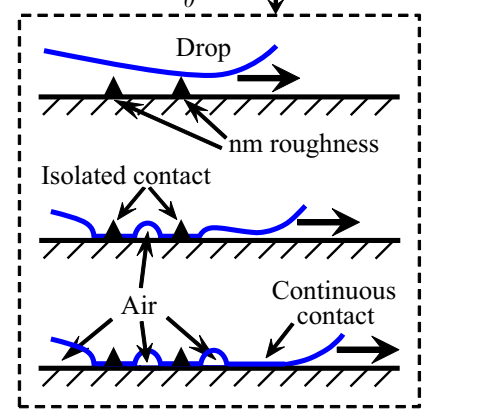

Top Isolated Continuous
view contact (b)
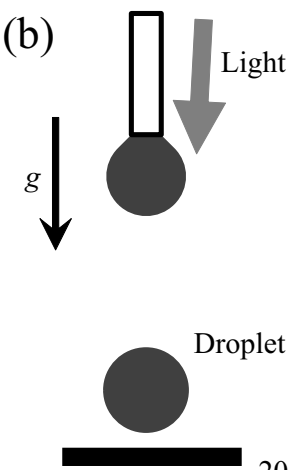

Droplet

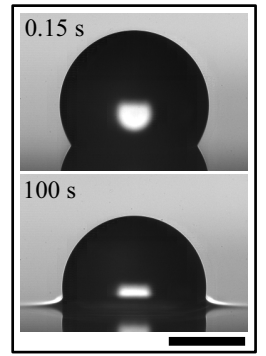

Glass slide, Mica or 20 million cSt silicone oil

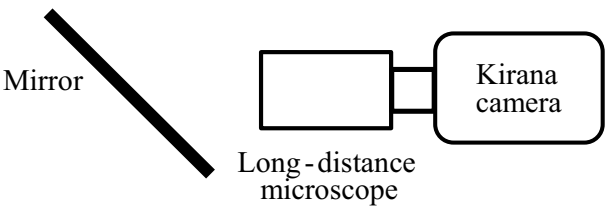

(c)

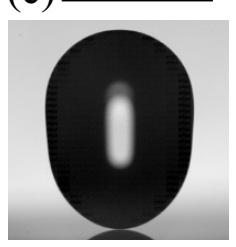

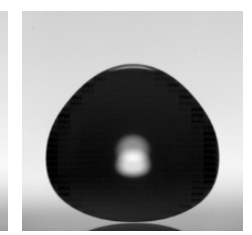
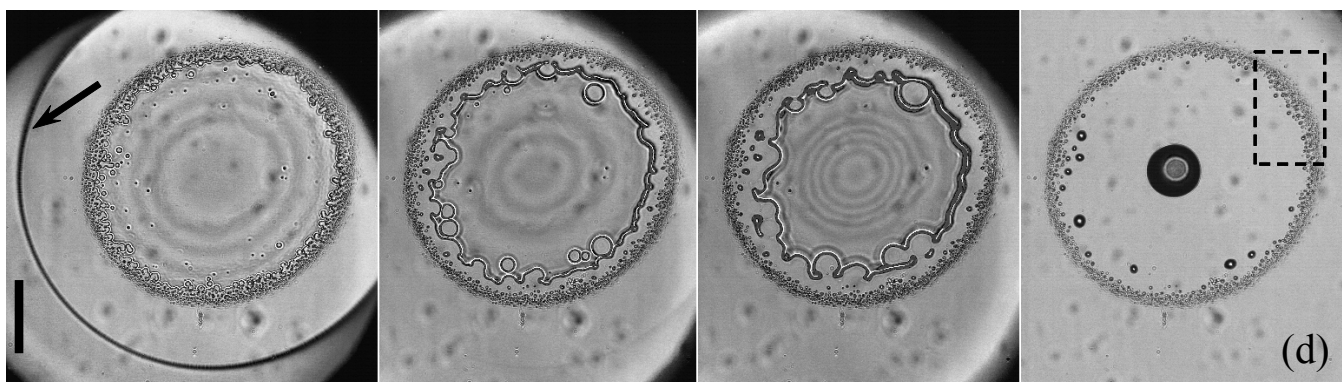

FIGURE 1. (a) Definition sketch, identifying the air-layer thickness $h(r)$ and the radial velocity $U$ of the kink at the outer edge of the dimple. Including at bottom, two schematics of the isolated contacts occurring before the fully wetted region, leading to the entrapment of micro-bubbles, from the side-view and top-view. (b) Sketch of the experimental setup, showing the imaging through the substrate. The arrangement of the laser-diodes with respect to the drop and video camera. Inset: The shape of a small water drop gently deposited on top of 20 million cSt silicone oil surface, to measure the contact angle, shown 0.15 and 100 seconds after contact. Scale bar is $500 \mu \mathrm{m}$. (c) Example shapes of the large water-drop at impact. Scale bar is $5 \mathrm{~mm}$. (d) Typical micro-bubbles marking the initial contact of a water-drop impacting a dirty (as delivered un-cleaned) glass micro-scope slide. Frames shown at 2, 7, $12 \& 400 \mu$ s from first contact, for $R_{b}=7.3 \mathrm{~mm}$ and $V=4.59 \mathrm{~m} / \mathrm{s}$, giving $W e=755, S t=5.8 \times 10^{-7}$ and $\epsilon^{-1}=22$. Scale bar is 200 $\mu \mathrm{m}$ long. The arrow in the first panel identifies the outer contact line. The dotted rectangle in the last panel identifies the approximate location of the viewing area in Fig. 3(a), for a different impact on cleaned glass. 


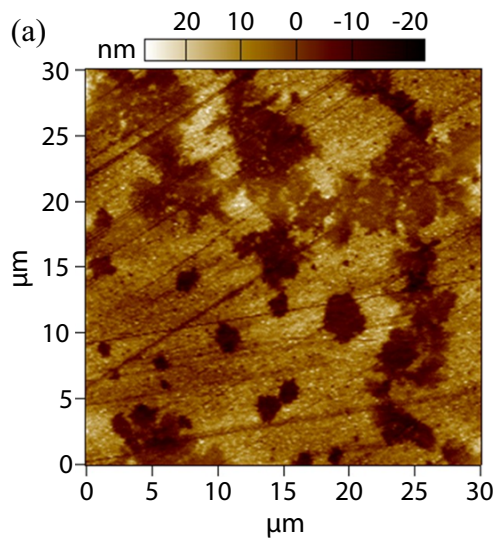

(b)

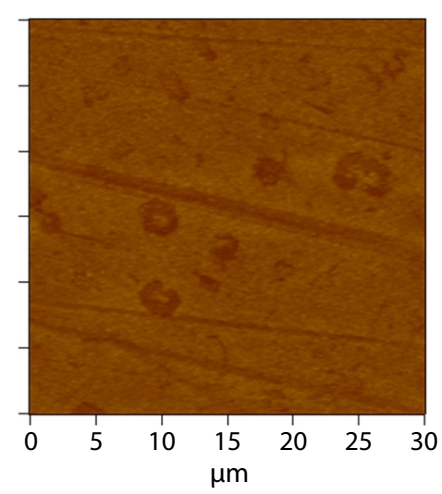

(c)

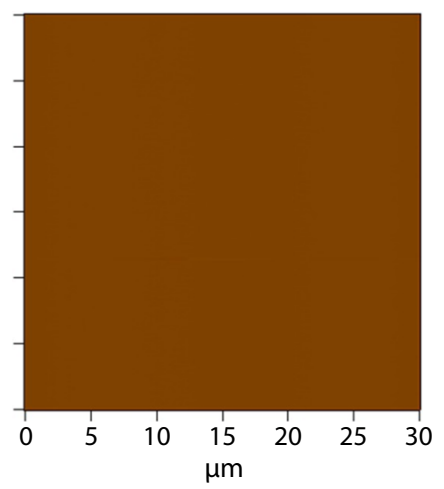

FiguRE 2. Roughness of the glass and mica surfaces. AFM topographic images of a $30 \times 30 \mu \mathrm{m}$ regions of (a) Corning glass, (b) Fisher glass and (c) freshly cleaved mica surface. Corning glass RMS $=7.3 \mathrm{~nm}$; Max to Min $=49 \mathrm{~nm}$; Fisher glass RMS $=1.2 \mathrm{~nm}$; Max to Min $=10 \mathrm{~nm}$; Mica RMS $<0.5 \mathrm{~nm}$. Supplemental materials show scans over larger surface areas.

new level of complexity in this fundamental process, which can only be revealed by the fastest imaging ever performed of this process.

\section{Experimental Setup}

\subsection{Optical and imaging setup}

The experimental configuration is sketched in Fig. 1(b) and is essentially the same as in Li \& Thoroddsen (2015), with imaging through the bottom glass plate. We use transmitted-light or reflective interferometry to measure the thickness of the air layer $h(r)$, while allowing imaging of the contact region. The pulsed monochromatic illumination $\left(\lambda_{\text {light }}=640 \mathrm{~nm}\right)$ is produced by 180 laser-diodes (SI-LUX640), one per video frame. This gives a depth resolution of $\lambda_{\text {light }} / 4=160 \mathrm{~nm}$, between adjacent dark and bright fringes. We use long-distance microscopes, primarily a Leica (Z16 APO), with pixel resolution down to $1.04 \mu \mathrm{m} / \mathrm{px}$. The rapid surface motions during first contact require ultra-high-speed video, acquired with the Kirana-05M from Specialized Imaging in Tring, UK (Crooks et al. (2013)), at frame rates up to 5 million fps. The extreme $200 \mathrm{~ns}$ time-resolution, allows us to follow individual fringes between video frames and eliminates some of the ambiguity at lower frame-rates, which has required color interferometry to get absolute layer thicknesses (van der Veen et al. (2012), de Ruiter et al. (2012)). Thickness profiles with multiple local minima, as well as rebounding non-contact cases will still need the two-colour technique to get absolute thicknesses (de Ruiter et al. (2012)).

Few experiments were performed with even larger optical magnification by using a $50 \times$ Mitutoyo objective, for a pixel resolution of $0.566 \mu \mathrm{m}$. The resulting images were not as clear owing to diffraction as well as the very narrow depth of focus (see Fig. 5).

The water drops used herein have equivalent diameter $D \simeq 5.2 \mathrm{~mm}$, which is significantly larger than the capillary length $a=\sqrt{\sigma /(\Delta \rho g)} \simeq 2.7 \mathrm{~mm}$, therefore showing large shape oscillations, which must be determined at impact. This is accomplished with sideviews using a Phantom V1610 CMOS video camera. Typical drop shapes are shown in Fig. 1(c). The impact velocities presented herein are between $V=1.2-4.2 \mathrm{~m} / \mathrm{s}$, corresponding to a range of Weber numbers $W e=\rho R V^{2} / \sigma=59-755$. 


\begin{tabular}{|c|ccc|}
\hline Fluid & $\begin{array}{c}\text { Density } \\
\rho\left[\mathrm{kg} / \mathrm{m}^{3}\right]\end{array}$ & $\begin{array}{c}\text { Viscosity } \\
\mu[\mathrm{cP}]\end{array}$ & $\begin{array}{c}\text { Surface tension } \\
\sigma[\mathrm{dyn} / \mathrm{cm}]\end{array}$ \\
\hline Air (A) & 1.2 & 0.0186 & - \\
Water (W) & 998 & 0.981 & 72.38 \\
Silicone oil 20 000 000 cSt & 970 & 19400000 & 21.2 \\
\hline
\end{tabular}

TABle 1. Properties of the fluids used in the experiments. Properties for silicone oil are taken from the suppliers data sheets.

\subsection{Glass and mica substrates}

We tested two different micro-scope-slide glass substrates, supplied by Corning (2947$75 \times 50)$ and Fisher Scientific. The glass slides were cleaned in a sequence of ultrasonic baths of acetone, isopropanol and deionized water for $10 \mathrm{~min}$ each. Following this treatment, the contact angle of water on the glass was about $20^{\circ}$. Figure $1(\mathrm{c})$ shows an impact onto dirty glass slide, i.e. one which has not been cleaned, showing much stronger air entrapment than for cleaned glass in what follows.

We also tested thin mica sheets which were attached to a glass slide with epoxy glue. The sheets were cleaved after every few drop impacts, to produce a fresh surface. This was done by depositing a Scotch tape onto the surface and rapidly peeling it off. A clear sign of successful peeling was that the tape would become smooth to the touch and loose its stickiness. Between drop impacts the surface was dried by blowing dry nitrogen, thereby removing any droplets or wet spots. Water fully wets the freshly cleaved mica.

Figure 2 shows atomic force measurements (AFM) of the surface roughness of the three different surfaces. The two glass slides have differing roughness, with the mica surface perfectly smooth within the measurements accuracy.

\subsection{Ultra-viscous liquid substrate}

In addition to water drop impacts onto the glass substrates, described above, we also imaged the impacts onto an extremely viscous liquid surface, immiscible to the water drop. Here the idea is to use a liquid to eliminate any surface roughness or asperities. For this purpose we use a silicone oil of 20 million cSt (Clearco Products). Properties are given in Table 1. This liquid is so viscous, that it takes hours to flatten, under gravity, after it is scooped onto a solid surface. We therefore deposit a fixed weight (2 g) of the material onto a glass plate, which we then heat to $80^{\circ} \mathrm{C}$ in an oven to make it spread over-night into a $1 \mathrm{~mm}$ thick circular patch. The contact angle of the water drop gently deposited on the immiscible silicone oil was measured, $150 \mathrm{~ms}$ after contact, to be $\simeq 118^{\circ}$, see inset in Fig. 1b. Contact angles are not well defined here as the triple line locally deforms the viscous surface (see Style et al. (2013)).

\section{Results}

\subsection{Effect of glass roughness vs smooth mica}

Figure 3(a) shows a close-up view of the outer edge of the air-disc, as it makes contact with the rougher glass (Corning). For orientation, see the approximate rectangle in Fig. 1(d), marked on a different impact. The second panel reveals isolated circular contacts, which occur slightly behind the continuous contact region which forms when the air-film thickness goes below some critical value. This region becomes visible in the third panel, see sketch in Fig. 1(a). The localized contacts grow in size and merge leaving microbubbles 

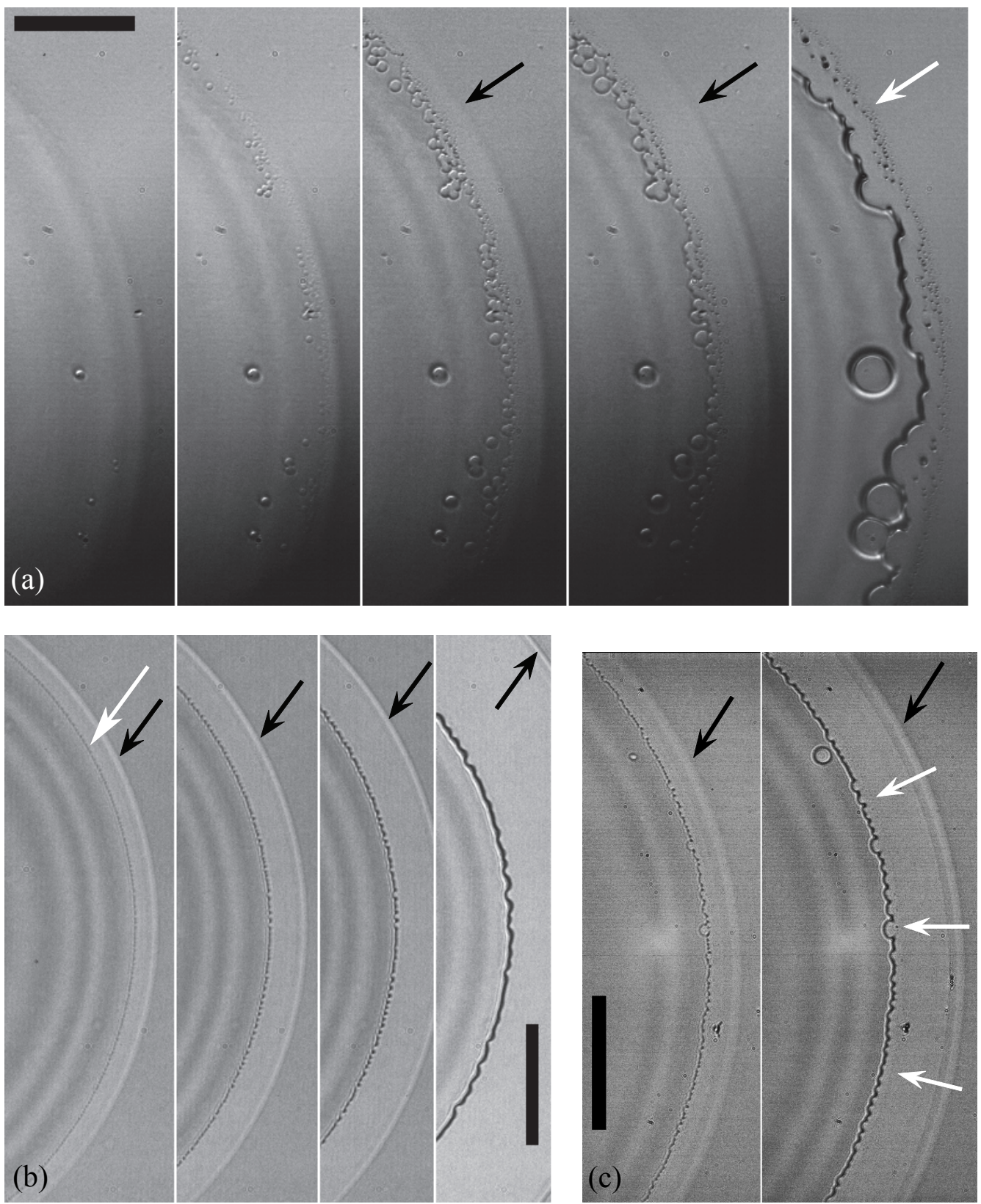

Figure 3. Comparison of the first contact of drops impacting on glass and mica surfaces. (a) Examples of ring of microbubbles at outer edge of the central contact on Corning glass, for water drop $D=5.2 \mathrm{~mm}, R_{b}=8.2 \mathrm{~mm}$ and impact velocity $V=2.43 \mathrm{~m} / \mathrm{s}$, giving $S t=9.2 \times 10^{-7}$ $\left(\epsilon^{-1}=5.98, W e=212\right)$. Frames are shown at $t=-0.2,0,0.2,0.4 \& 1.8 \mu \mathrm{s}$ around first contact. The white arrow points out the ring of micro-bubbles left behind. (b) Impact on a molecularly smooth freshly cleaved mica surface, for $R_{b}=4.1 \mathrm{~mm}$ and impact velocity $V=2.10 \mathrm{~m} / \mathrm{s}\left(S t=2.1 \times 10^{-6}\right.$, $\left.\epsilon^{-1}=3.37, W e=158\right)$, shown at $t=0,0.2,0.4 \& 1.6 \mu$ s from first contact. The white arrow points at the inner contact line. No micro-bubbles are left behind. (c) Impact on cleaned Fisher micro-scope slide, shown at $t=0,0.4 \mu \mathrm{s}$ for $R_{b}=5.1 \mathrm{~mm}, V=2.33 \mathrm{~m} / \mathrm{s}\left(S t=1.5 \times 10^{-6}, \epsilon^{-1}=4.6\right.$, $W e=195)$. The scale bars are all $100 \mu \mathrm{m}$ long. White arrows, in last panel, point out a faint ring of tiny bubbles, which can be better observed in an enhanced supplemental image and video. The black arrows in all panels indicate the outer edge of the fully wetted contact. 
between them and the fully wetted outer region, the outer edge of which is marked with black arrows. Immediately following this, a rugged inner contact line forms and starts contracting into the central bubble. The fine dark spots left behind are the microbubbles, with typical size of $2-5 \mu \mathrm{m}$. Figure $3(\mathrm{~b})$ contrasts this with the impact on a molecularly smooth mica sheet. Here the initial contact appears uniformly around the periphery, with no obvious isolated circular contacts before continuous wetting. The contraction of the thin edge produces fine undulations in the azimuthal direction, but no isolated microbubbles are left behind, during the retraction of the inner contact line. Similar influence of roughness is observed for other impact conditions, for impact velocities larger than the skating regime, i.e. for $S t<6 \times 10^{-6}$ or $W e<70$. The AFM measurements in Fig. 2 show that the Fisher glass is much smoother than the Corning glass, as is clearly evident in Fig. 3(c), where the contact is similar to that with the mica and less than a handful of clearly identifiable local contacts are observed, leaving only 1 distinct microbubble. However, a fine ring of tiny micro-bubbles (white arrows) can be discerned from a careful inspection of the video, shown in supplemental material where we include two enhanced frames. In this case, the localized contacts and formation of the fully wetted region, must occur within the 200 ns interframe time.

Consistent with this picture, Fig. 1(d) showed much larger number of entrapped microbubbles for impact on a rougher uncleaned glass surface.

\subsection{Impact on ultra-viscous liquid substrate}

In a further attempt to reduce surface roughness, we also tried impacts on an ultraviscous liquid film. Using a liquid surface one can minimize any asperities, other than possible dust settling on the surface. In Fig. 4(d) we first compare the thickness profiles $h(r)$ of the air-layers for impacts of identical water drops onto Corning glass vs the ultraviscous $20 \times 10^{6} \mathrm{cSt}$ silicone oil. One striking difference between these two substrates, is the greatly reduced compression of the gas, in the air-disc, for impacts on the viscous surface. The air-disc on the ultra-viscous substrate is twice as thick and 1.5 times larger in diameter. This is evident from the reduced expansion of the layer after first contact. Clearly, the compliance of the ultra-viscous substrate reduces the maximum pressure and this is a testament to the large compression which occurs (Liu et al. (2013); Li \& Thoroddsen (2015)). This is further demonstrated during the subsequent rapid expansion of the air layer, which is observed for the impact on the solid Corning glass surface, is almost eliminated by the viscous substrate. Here the more compressed air on the glass expands at the centerline by a factor of 2.3 from $1.12 \rightarrow 2.56 \mu \mathrm{m}$, while on the silicone oil it expands by only a factor of 1.25 from $2.56 \rightarrow 3.2 \mu \mathrm{m}$. Here the added inertia of the substrate plays no role, like for identical liquids (Bouwhuis et al. (2015)).

Microbubbles are also left behind for this water-on-silicone-oil configuration. However, they arise not from localized contacts like in Fig. 3(a), rather from an azimuthal instability of the rapidly moving triple line, as shown in Fig. 4(b). The wavelength $\lambda$ of this instability follows quite well the local thickness $h(r)$ of the air film (Fig. 4c), with $\lambda \simeq 120 h(r)$. Here, $h(r)$ is determined from the interference fringes at first contact, as shown in the inset. The wavelength grows by merging of adjacent crests, while their tips shed microbubbles which become progressively larger. Similar bubble shedding is observed for thin air-layers in Mesler entrainment, see figure 4 in Thoroddsen et al. (2012).

The contraction of the inner edge of the air-disc starts out at $u_{\text {edge }}=10.1 \mathrm{~m} / \mathrm{s}$, corresponding to a capillary number based on the water properties, of $C a=\mu u_{e d g e} / \sigma=$ 0.14. The edge velocity slows as the edge thickens and the pinch-off of bubbles stops, after $13 \mu \mathrm{s}$, when $u_{e d g e} \simeq 3.45 \mathrm{~m} / \mathrm{s}$, corresponding to $C a=0.048$. Regular undulations 
(a)
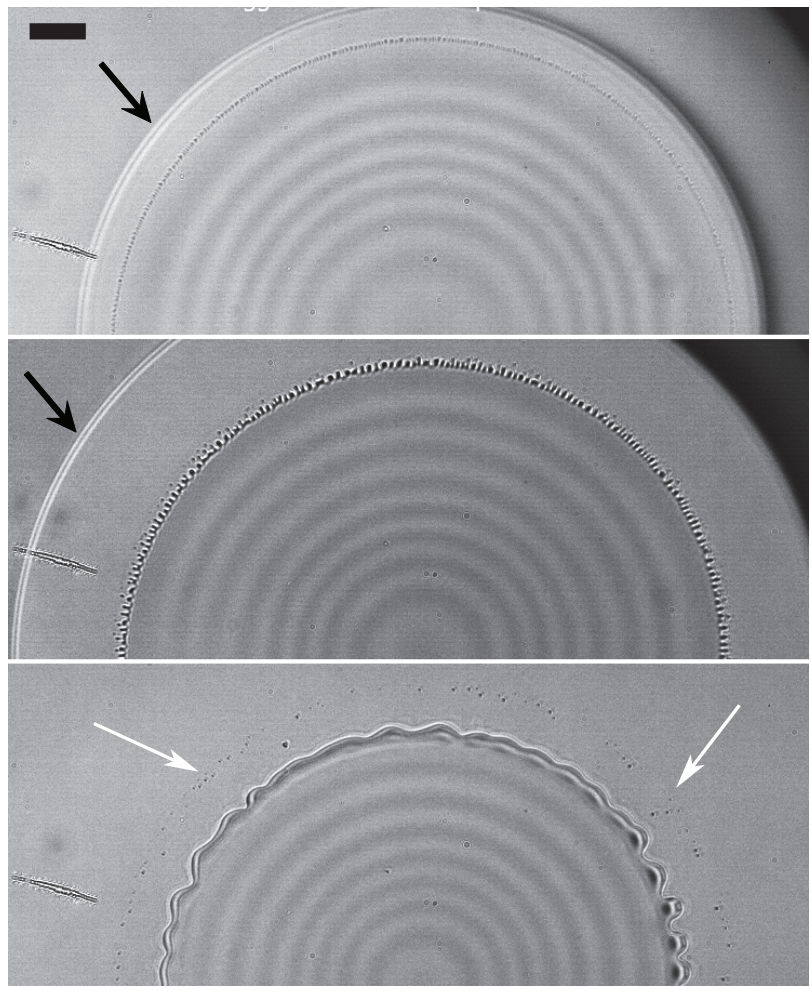

(b)

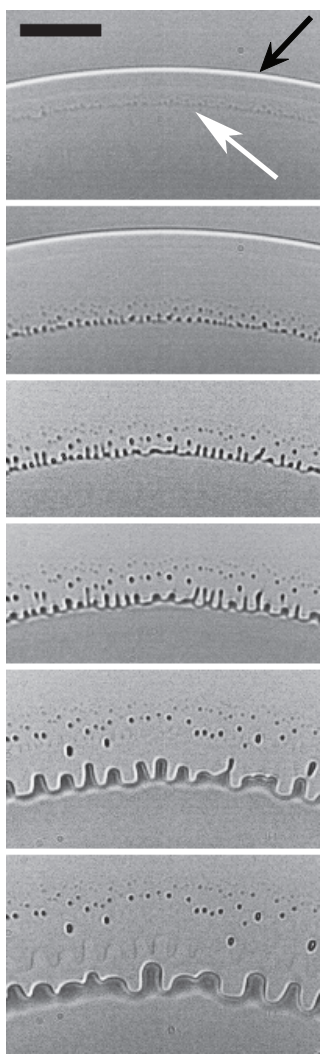

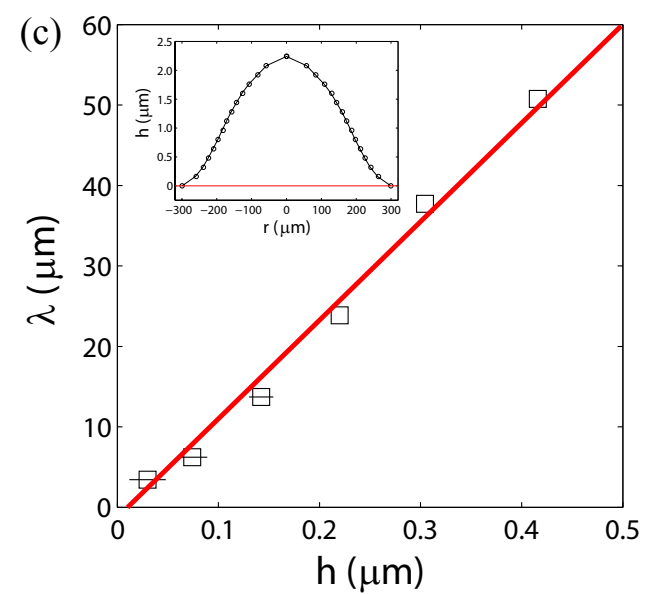

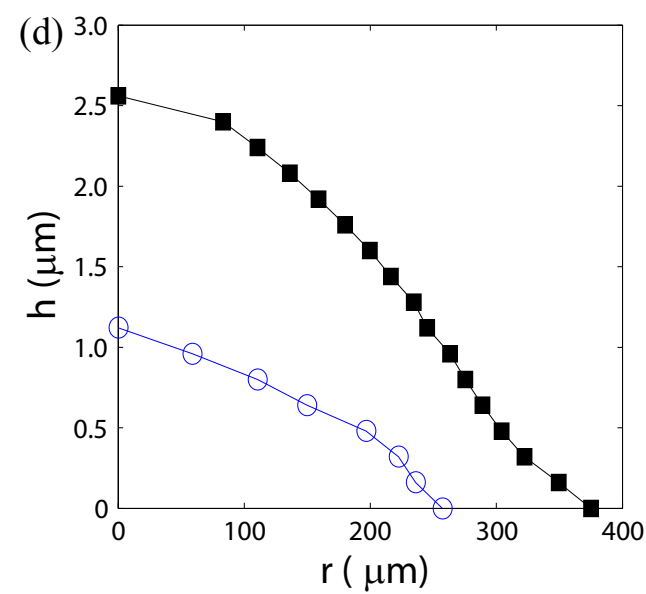

Figure 4. (a) The air-disc under a water drop impacting on a $20 \times 10^{6} \mathrm{cSt}$ silicone oil surface, at velocity $V=3.15 \mathrm{~m} / \mathrm{s}$ and $R_{b}=2.4 \mathrm{~mm}$. Frames are shown at $t=0.4,1.8 \& 12.2 \mu \mathrm{s}$ after first contact $\left(S t=2.4 \times 10^{-6}, \epsilon^{-1}=7.5, W e=356\right)$. The white arrows point out entrapped micro-bubbles. Black arrows in all panels identify the outer contact line. (b) Undulations and pinch-off of microbubbles at the contracting edge of the air-disc, shown at $t=0,1,2,3,7.5 \&$ $12.5 \mu \mathrm{s}$. Scale bars are $50 \mu \mathrm{m}$. White arrow in first panel identifies the inner contact line. (c) The azimuthal wavelength of the characteristic undulations at the edge of the contracting triple-line, versus the initial local thickness of the air-film, shown in the inset. (d) Comparison of the air-film thickness profile for identical water drops $\left(V=3.10 \mathrm{~m} / \mathrm{s} ; R_{b}=4.0 \mathrm{~mm} ; S t=1.5 \times 10^{-6}, \epsilon^{-1}=8.3\right.$, $W e=344$ ) impacting a Corning glass surface (open circles) and $20 \times 10^{6}$ cSt silicone oil (filled squares). 
(a)

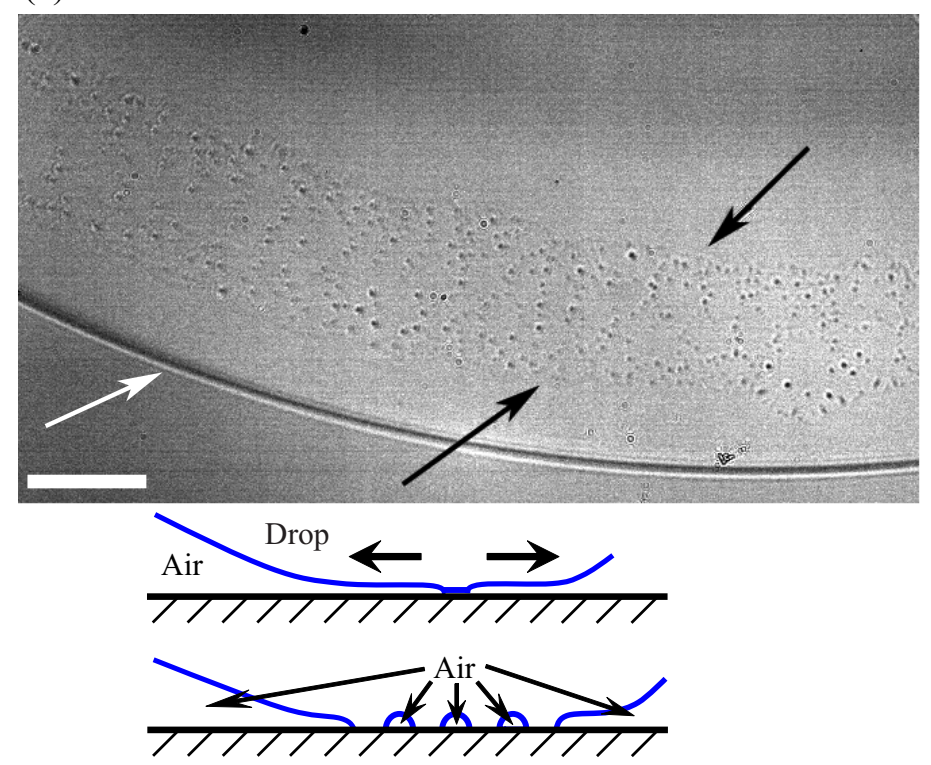

(b)

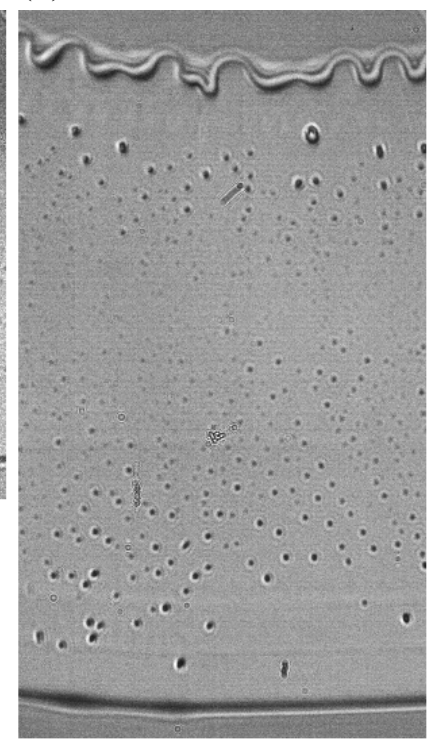

FIGURE 5. (a) Example of the rupture of the thin air-film, under the gliding edge, for water on $20 \times 10^{6} \mathrm{cSt}$ silicone oil, during low impact velocity $U=1.28 \mathrm{~m} / \mathrm{s}, R_{b}=4.5 \mathrm{~mm}\left(S t=3.2 \times 10^{-6}\right.$, $\left.\epsilon^{-1}=1.10, W e=59\right)$. The two dark arrows point out the edges of the rupture zone, which is a significant distance behind the outer moving edge of the gliding marked by a white arrow. Scale bar is $50 \mu \mathrm{m}$ long. (b) The band of micro-bubbles $11 \mu \mathrm{s}$ after the start of ruptures in (a), when the entrapment of microbubbles has stopped and the rupture front has caught up with the outer edge of the thin air layer.

have here essentially disappeared when the edge has contracted to the radius where the original thickness was $h(r) \simeq 0.5 \mu \mathrm{m}$.

The nature of this long-wavelength instability is unclear. The very long wavelength, compared to the layer thickness, appears to rule out a capillary Rayleigh instability of a thicker edge. Furthermore, $\lambda$ is orders of magnitude smaller than the radius of the air-disc, so we conclude that the azimuthal curvature of the triple-line is not important. Keep in mind that the presence of the triple-line, between water and high-viscosity liquid, has strong effect on its contraction, greatly slowing it down (Li et al. (2014)).

\subsection{Low impact velocity, skating and film ruptures}

For very low impact velocities, it has been shown that drops can skate on a thin layer of air (Kolinski et al. (2012)) and even rebound without touching the substrate (de Ruiter et al. (2014), Kolinski et al. (2014)). Such skating of the outer edge on a thin layer of air is indeed observed herein for low impact velocities. In the wateron-20-million-cSt configuration, this occurs when the $S t>\sim 5 \times 10^{-6}$, corresponding to $W e<70$. Keep in mind that this is for much larger $W e$ than the rebounding regime studied in de Ruiter et al. (2014). In our impact-velocity range, these films rupture and we do not look at rebounding. Figure 5 and supplemental video, show such a low impact velocity case for water drop on $20 \times 10^{6}$ cSt silicone film. This thin gliding air-layer then ruptures simultaneously at multiple locations around the periphery, merging into a band of ruptures, as marked by the arrows in Fig. 5(a). The original location of the ruptures is $\sim 50 \mu \mathrm{m}$ behind the outer edge of the thin air-layer. The edge-speed of the growing 
wet spots is measured at $\simeq 15 \mathrm{~m} / \mathrm{s}$, which is faster than the contracting edge in Fig. 4, which indicates layer-thickness much thinner than $100 \mathrm{~nm}$. Numerous micro-bubbles are entrapped where these ruptures meet, thereby forming a band of contacts, which widens rapidly in the radial direction. The outer edge of this rupture zone moves faster than the edge of the air-layer marking the spreading drop (white arrow in Fig. 5) and catches up with it after $9 \mu \mathrm{s}$, in this realization. This eliminates the skating of the edge and the accompanying micro-bubble entrapment, leaving behind a $200 \mu \mathrm{m}$ wide band of bubbles. The total number of visible micro-bubbles in this entire band around the full periphery is here estimated to be $\sim 12800$, ranging in sizes from $1-5 \mu \mathrm{m}$. The bubble sizes are smallest near the first rupture, where the air layer should be thinnest (Beilharz et al. (2015)) and grow larger towards the edges of the band (Fig. 5b). Such bands of bubbles have been observed for impacts of viscous drops in Thoroddsen et al. $(2005,2010)$ and Palacios et al. (2012).

\section{Discussion and conclusions}

Herein, we have used ultra-fast video imaging to observe the first contact of an impacting drop with a solid or an extremely viscous substrate. Our results show that $\sim 10 \mathrm{~nm}$ surface roughness promotes localized contacts slightly before full wetting is established, thereby leaving behind the previously observed ring of microbubbles (Thoroddsen et al. (2005)). Impacts on smoother glass reduces the sizes of these bubbles and none are visible for impacts onto the molecularly smooth mica substrate, at our optical resolution. However, the first contact on the mica shows fine azimuthal undulations, the origins of which remains a mystery, but we suggest is due to contact line dynamics.

Impacts onto the ultra-viscous silicone surface also leave a ring of microbubbles, not due to roughness, but through an azimuthal instability at the triple line, by tip streaming.

Mandre \& Brenner (2012) theory (their fig. 8a) presents the roughness needed to interfere with the lubricating air-film for different impact conditions. For our water drop sizes and impact velocities, the very small roughness of the freshly-cleaved mica sheets should be insufficient to break the lubricating films in Fig. 3(b). This implies that some phenomenon which is left out of their skating model is at play. We suggest the ruptures are due to van der Waals forces, when the air-film becomes significantly thinner than $100 \mathrm{~nm}$, which we cannot measure with our optical setup. The fact that ruptures arise simultaneously along the periphery and at the same radial distance, in Fig. 5(a), rules out isolated larger asperities as the trigger.

Furthermore, even for molecularly smooth mica surfaces the continuous contact shows that the skating solutions are not realized above a moderate impact velocity.

\section{Acknowledgements}

The work reported herein was funded by King Abdullah University of Science and Technology (KAUST). The AFM imaging was performed in the KAUST Microfluidics Thrust Area Labs.

\section{REFERENCES}

Beilharz, D., Guyon, A., Li, E. Q., Thoraval, M.-J. \& Thoroddsen, S. T. Antibubbles and fine cylindrical sheets of air, J. Fluid Mech., 779, 87-115 (2015).

Bouwhuis W., van der Veen, R. C. A., Tran T., KeiJ, D. L., Winkels, K. G., Peters, 
I. R., van der Meer D., Sun, C., Snoeijer J. H. \& Lohse, D. 2012. Maximal air bubble entrainment at liquid-drop impact. Phys. Rev. Lett., 109, 264501.

Bouwhuis W., Hendrix, M. H. W., van der Meer D. \& Snoeijer J. H. 2015. Initial surface deformations during impact on a liquid pool. J. Fluid Mech., 771, 503-519.

Chandra, S. \& Avedisian, C. T. 1991. On the collision of a droplet with a solid surface. Proc. R. Soc. Lond. A, 432, 13-41

Crooks, J., Marsh, B., Turchetta, R., Taylor, K., Chan, W., Lahav, A. \& Fenigstein, A. 2013 Kirana: a solid-state megapixel uCMOS image sensor for ultrahigh speed imaging. Proc. SPIE, 8659, 865903.

De Ruiter, J., Lagraauw, R., van Den Ende, D \& Mugele, F. 2014 Wettability-independent bouncing on flat surfaces mediated by thin air films. Nat. Phys. 11, 48-53.

de Ruiter, J., Оh, J. M., van Den Ende, D. \& Mugele, F. 2012 Dynamics of collapse of air films in drop impact. Phys. Rev. Lett., 108, 074505.

Driscoll, M. M. \& NAGEL, S. R. 2011 Ultrafast interference imaging of air in splashing dynamics. Phys. Rev. Lett., 107, 154502.

Duchemin, L. \& Josserand, C. 2011 Curvature singularity and film-skating during drop impact. Phys. Fluids 23, 091701.

Hicks, P. D., Ermanyuk, E. V., Gavrilov, N. V. \& Purvis, R. 2012. Air trapping at impact of a rigid sphere onto a liquid. J. Fluid Mech. 695 310-320

Hicks, P. D. \& Purvis, R. 2010. Air cushioning and bubble entrapment in three-dimensional droplet impacts. J. Fluid Mech. 649, 135-163

Hicks P. D. \& PuRvis R. 2013. Liquid-solid impacts with compressible gas cushioning, J. Fluid Mech., 735, 120-149.

Korobkin, A. A., Ellis, A. S. \& Smith, F. T. 2008. Trapping of air in impact between a body and shallow water. J. Fluid Mech., 611, 365-394

Kolinski, J. M., Mahadevan, L. \& Rubinstein, S. M. 2014 Drops can bounce from perfectly hydrophilic surfaces. Euro. Phys. Lett. 108, 24001.

Kolinski, J. M., Rubinstein, S. M., Mandre, S., Brenner, M. P., Weitz, D. A. \& Mahadevan, L. 2012 Skating on a film of air: drops impacting on a surface. Phys. Rev. Lett., 108, 074503.

Lee, J. S., Weon, B. M., Je, J. H. \& FezzaA, K. 2012 How does an air film evolve into a bubble during drop impact? Phys. Rev. Lett., 109204501.

Li, E. Q., Al-Otaibi, S., Vakarelski, I,. U., Thoroddsen, S. T. 2014 Satellite formation during bubble transition through an interface between immiscible liquids, J. Fluid Mech., 744, R1.

LI, E. Q. \& Thoroddsen, S. T. 2015 Time-resolved imaging of a compressible air disc under a drop impacting on a solid surface, J. Fluid Mech., 780, 636-648.

LiU, Y., TAN, P. \& XU, L. 2013 Compressible air entrapment in high-speed drop impacts on solid surfaces. J. Fluid Mech., 716, R9.

Mandre, S. \& Brenner, M. P. 2012. The mechanism of a splash on a dry solid surface, J. Fluid. Mech., 690, 148-172

Mandre, S., Mani, M. \& Brenner, M. P. 2009. Precursors to splashing of liquid droplets on a solid surface. Phys. Rev. Lett., 102, 134502.

Mani, M., Mandre, S. \& Brenner, M. P. 2010. Events before droplet splashing on a solid surface. J. Fluid. Mech., 647, 163-185.

Palacios, J., Hernández, J., Gómez, P., Zanzi, C., López, J. 2012 On the impact of viscous drops onto dry smooth surfaces, Exp. Fluids, 52, 1449-1463.

Sмith, F. T., Li, L. \& Wu, G. X. 2003. Air cushioning with a lubrication/inviscid balance. J. Fluid Mech., 482, 291-318.

Style, R. W., Hyland, C., Boltyanskiy, R., Wettlaufer, J. S. \& Dufresne, E. R. 2013 Surface tension and contact with soft elastic solids. Nat. Comm., 4, 2728.

Thoroddsen, S. T., Eтоh, T. G., Takehara, K., Ootsuka, N. \& Hatsuki, Y. 2005. The air-bubble entrapped under a drop impacting on a solid surface. J. Fluid Mech., 545, 203-212.

Thoroddsen, S. T. \& Sakakibara, J. 1998. Evolution of the fingering pattern of an impacting drop. Phys. Fluids, 10, 1359-1374. 
Thoroddsen, S. T., Takehara, K. \& Etoh, T. G. 2010. Bubble entrapment through topological change. Phys. Fluids, 22, 051701

Thoroddsen, S. T., Thoraval, M.-J., Takehara, K. \& Etoh, T. G. 2012 Micro-bubble morphologies following drop impacts onto a pool surface, J. Fluid Mech., 708, 469-479.

van der Veen, R. C. A., Tran, T., Lohse, D. \& Sun, C. 2012 Direct measurements of air layer profiles under impacting droplets using high-speed color interferometry. Phys. Rev. E 85, 026315 . 